\title{
CONTRIBUCIÓN AL CONOCIMIENTO DE LOS CRISÓPIDOS DE COQUIMBO, PATAGONIA Y TIERRA DEL FUEGO (ARGENTINA, CHILE) (INSECTA, NEUROPTERA, CHRYSOPIDAE)
}

\author{
V. J. Monserrat* y S. de Freitas**
}

\begin{abstract}
RESUMEN
Se anotan nuevos datos sobre la morfología, distribución y biología de cinco especies de crisópidos (Insecta, Neuroptera, Chrysopidae) capturadas durante los muestreos realizados en Patagonia, Tierra del Fuego y Coquimbo (Argentina, Chile). Se describen los estadios juveniles y los adultos de Ungla argentina (Navás, 1911), Ungla binaria (Navás, 1922) y Chrysopodes (Neosuarius) porterina (Navás, 1910). Esta última especie y Chrysoperla defreitasi Brooks, 1994 se citan por primera vez de Argentina.

Palabras clave: Faunística, estadios juveniles, planta substrato, Insecta, Neuroptera, Chrysopidae, Patagonia, Coquimbo, Argentina, Chile.
\end{abstract}

\section{SUMMARY}

A contribution to the knowledge of the green-lacewings from Coquimbo, Patagonia and Tierra del Fuego (Argentina, Chile) (Insecta, Neuroptera, Chrysopidae)

New data on the morphology, distribution and biology of five species of green-lacewings (Insecta, Neuroptera, Chrysopidae) collected from samplings made in Patagonia, Tierra del Fuego and Coquimbo (Argentina, Chile) are given. The general morphology and the preimaginal stages of Ungla argentina (Navás, 1911), Ungla binaria (Navás, 1922) and Chrysopodes (Neosuarius) porterina (Navás, 1910) are described. This last species and Chrysoperla defreitasi Brooks, 1994 are recorded from Argentina for the first time.

Key Words: Faunistics, Juvenile Instars, Plant substrate, Insecta, Neuroptera, Chrysopidae, Patagonia, Coquimbo, Argentina, Chile.

\section{Introducción}

La familia Chrysopidae (Insecta, Neuroptera) incluye unas especies 1.200 especies (Brooks \& Barnard, 1990) y es una de las familias más interesantes dentro del orden Neuroptera debido a su amplia y casi cosmopolita distribución geográfica, al elevado número de ejemplares que con frecuen- cia constituyen sus poblaciones y, especialmente, por ser muy importantes agentes de control biológico sobre pequeños fitófagos (Canard et al., 1984; Mc Ewen et al., 2001).

La taxonomía y sistemática de esta familia ha sido recientemente tratada a nivel de género por Brooks \& Barnard (1990) quienes aportan valiosos datos que posibilitan una visión general muy deta-

* Dept. de Zoología y Antropología Física, Facultad de Biología, Universidad Complutense, 28040 Madrid (España). E-mail: artmad@bio.ucm.es

** Dept. de Fitossanidade, FCAV/UNESP, Via de Acesso Prf. Paulo Donato Castellane s/n, 14. 884-900 Jaboticabal, São Paulo (Brasil). E-mail: serfre@fcav.unesp.br 
llada sobre la taxonomía, sistemática, filogenia y biogeografía de esta familia. Sin embargo aún queda mucho por hacer, ya que existen enormes áreas geográficas muy deficientemente muestreadas y quedan grandes lagunas sobre la morfología, variabilidad, taxonomía, biología, faunística y estadios juveniles de la mayoría de las especies conocidas.

Con referencia a la Fauna Neotropical, que venía estando bastante olvidada en relación a otras áreas biogeográficas, existen multitud de antiguas descripciones y citas dispersas en la bibliografía y han sido recientemente publicados nuevos trabajos que han aportado nuevos datos y un mayor conocimiento sobre la biología, taxonomía, morfología, distribución, fenología, biología y estadios juveniles de numerosas especies de esta familia en Centro y Sudamérica. Se han publicado listados, catálogos, nuevos taxones y la revisión de varios géneros de crisópidos perteneciente a la Fauna Neotropical, así como la fauna de crisópidos de algún determinado país, como es el caso de Costa Rica (Kimmins, 1952; Adams, 1967, 1969, 1975, 1977, 1978a, b, 1979, 1982a, b, 1983, 1985, 1987; Penny, 1977, 1996, 1997, 2002; González Olazo, 1987; Adams \& Penny, 1986, 1987, 1992; Freitas \& Fernández, 1992; Penny \& Lee, 1996; Carvalho et al., 1998; Auad et al., 2001a, b, 2002; Freitas \& Penny, 2001a, b; Gitirana Neto et al., 2001; Penny \& Freitas, 2001; Perez Gelabert \& Flint, 2001; Freitas, 2003, etc.), que han contribuido a mejorar notablemente el conocimiento sobre la fauna de crisópidos de la Región Neotropical.

De estos trabajos se desprende la existencia de cierta información sobre los crisópidos de Coquimbo, pero sobre todo se evidencia la escasa información que disponemos sobre la fauna de crisópidos de Patagonia y de Tierra del Fuego. Aumentar el nivel de conocimiento que poseemos sobre los Chrysopidae de estas zonas y conocer su límite de distribución, especialmente de esta enorme y particular extensión del cono sur americano (Patagonia y Tierra del Fuego) es el motivo principal de la presente contribución. En ella aportamos nuevos datos sobre la distribución geográfica, altitudinal, biología, morfología, estadios preimaginales y / o variabilidad de las especies que hemos encontrado durante los muestreos realizados.

\section{Material y Métodos}

Para la toma de datos los muestreos se realizaron en dos itinerarios, uno en la Región de Coquimbo (Chile) y otro en Patagonia y Tierra del
Fuego (Argentina y Chile), tratando con estos itinerarios de que los muestreos, dentro de nuestras posibilidades, cubrieran mayoritariamente la zona a estudiar, que barrieran la mayor superficie posible y que alcanzaran el extremo sur del continente, realizando paradas sucesivas para la toma de muestras (Monserrat, 2003, 2005).

De las 59 localidades muestreadas (6 en Coquimbo, Chile, y 53 en Patagonia y Tierra del Fuego, Argentina y Chile) se han hallado crisópidos en 13 de ellas. La ubicación administrativa, denominación, altitud sobre / bajo el nivel del mar y coordenadas geográficas de estas localidades se anotan ordenadas por su latitud creciente y numeradas en la lista adjunta:

\section{CHILE}

1: IV REGIÓN, COQUIMBO, La Serena, Punta Teatinos, 10 $\mathrm{m}, 71^{\circ} 17^{\prime} \mathrm{W}, 29^{\circ} 49^{\prime} \mathrm{S}$.

2: IV REGIÓN, COQUIMBO, Quebrada Honda, Elqui,

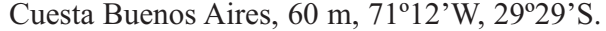

\section{ARGENTINA}

3: PROVINCIA DE BUENOS AIRES, Tornquist, Parque Tornquist, Garganta Olvidada, $500 \mathrm{~m}, 62^{\circ} 03^{\prime} \mathrm{W}, 38^{\circ} 03^{\prime} \mathrm{S}$.

4: PROVINCIA DE BUENOS AIRES, Tornquist, Cerro Tornquist, $400 \mathrm{~m}, 62^{\circ} 10^{\prime} \mathrm{W}, 38^{\circ} 04^{\prime} \mathrm{S}$.

5: PROVINCIA DE BUENOS AIRES, Bahía Blanca Ciudad, $20 \mathrm{~m}, 62^{\circ} 11^{\prime} \mathrm{W}, 38^{\circ} 44^{\prime} \mathrm{S}$.

6: PROVINCIA DE BUENOS AIRES, Villarino, Laguna de Chasicó-Estancia La Aurora, entre -24 y $-45 \mathrm{~m}, 62^{\circ} 55^{\prime} \mathrm{W}$, $38^{\circ} 45^{\prime} \mathrm{S}$.

7: PROVINCIA DE BUENOS AIRES, Médanos, Villarino, $25 \mathrm{~m}, 62^{\circ} 54^{\prime} \mathrm{W}, 38^{\circ} 46^{\prime} \mathrm{S}$.

8: PROVINCIA DE BUENOS AIRES, Pehuen Có, $10 \mathrm{~m}$, $61^{\circ} 37^{\prime} \mathrm{W}, 39^{\circ} 00^{\prime} \mathrm{S}$.

9: PROVINCIA DE BUENOS AIRES, Cabeza de Buey, 15 $\mathrm{m}, 62^{\circ} 30^{\prime} \mathrm{W}, 39^{\circ} 10^{\prime} \mathrm{S}$.

10: PROVINCIA DE BUENOS AIRES, Mayor Buratovich, 15 $\mathrm{m}, 62^{\circ} 37^{\prime} \mathrm{W}, 39^{\circ} 15^{\prime} \mathrm{S}$.

11: PROVINCIA DE RÍO NEGRO, Paso Chacabuco, Río Limay, $510 \mathrm{~m}, 71^{\circ} 02^{\prime} \mathrm{W}, 40^{\circ} 45^{\prime} \mathrm{S}$.

12: PROVINCIA DE BUENOS AIRES, Carmen de Patagones, Ribera Río Negro, $10 \mathrm{~m}, 62^{\circ} 59^{\prime} \mathrm{W}, 40^{\circ} 48^{\prime} \mathrm{S}$.

13: PROVINCIA DE RÍO NEGRO, N. El Bolsón, Los Repollos, Cerro Saturnino, $655 \mathrm{~m}, 71^{\circ} 25^{\prime} \mathrm{W}, 41^{\circ} 50^{\prime} \mathrm{S}$.

El material era capturado mangueando la vegetación arbórea y arbustiva existente en cada localidad y ocasionalmente se recolectó material atraído a la luz durante la noche. Algunos adultos fueron mantenidos en cajas de cultivo de 4 x 4 x $1 ' 5 \mathrm{~cm}$ a temperatura ambiente para la obtención de puestas y se les proporcionó humedad mediante un algodón humedecido en agua y ninfas e imagos de áfidos para su alimentación. Las puestas obtenidas y las larvas en desarrollo se mantuvieron en estas cajas de cultivo entre $15-24^{\circ} \mathrm{C}$ y tam- 
bién fueron alimentadas con ninfas e imagos de diferentes especies de áfidos.

Para la descripción de la morfología y quetotaxia larvaria los ejemplares se aclaraban previamente en ácido láctico. Los dibujos se han realizado con una lupa binocular Leica MZ12.5 con cámara fotográfica DC300 incorporada.

El material citado queda depositado en la colección de su recolector (V. J. Monserrat), ubicada en el Departamento de Zoología y Antropología Física de la Universidad Complutense de Madrid (España), salvo algunos ejemplares que se han depositado en la colección de S. de Freitas de la Universidade Estadual Paulista de São Paulo (SF) y en el Museo Argentino de Ciencias Naturales "Bernardino Rivadavia” de Buenos Aires (MACN).

Para cada una de las cinco especies citadas se recopilan las referencias bibliográficas existentes sobre su taxonomía, faunística, morfología y biología según las diferentes denominaciones con las que hayan sido citadas, así como las de sus conocidas sinonimias, anotándose los datos que se hayan publicado sobre sus alas (a), biología (biol), descripción original (des), distribución (dis), huevo (h), larvas (la), morfología (morf), pupa (pp), taxonomía (tax), genitalia masculina $\left(\sigma^{\top}\right)$ o femenina ( ) o aparezcan en claves (cl), listados (list), notas (nt) o referencias (ref).

También anotamos para cada una de ellas su distribución geográfica previamente conocida, las localidades donde han sido halladas, según la numeración anteriormente listada, las fechas de captura, el número de $\sigma^{\top} \sigma^{\top}$, 우 우 o larvas capturadas, el sustrato vegetal sobre el que han sido hallados y aquellos datos de interés sobre su biología, morfología, estadios preimaginales, comportamiento o longevidad que puedan contribuir a su mejor conocimiento. Para la sistemática y morfología se sigue la ordenación general dada por Brooks \& Barnard (1990) y para la quetotaxia larvaria de sigue a Tauber et al. (1998). Para la identificación de las especies de árboles y plantas citadas se han utilizado las obras de Hoffmann (1997), Erize (2000a, b) y Haene \& Gostaro (2001).

\section{Resultados}

De las 6 localidades de Coquimbo (Chile) y de las 53 localidades de Patagonia y Tierra del Fuego (Argentina y Chile) en las que se realizó la toma de muestras (Monserrat, 2003, 2005) se han encontrado 73 ejemplares pertenecientes a 5 especies de crisópidos en sólo 13 de estas localidades, todas ellas situadas por encima del paralelo $41^{\circ} 50^{\prime} \mathrm{S}$ y no se ha obtenido ningún dato en Patagonia más al sur, ni en la parte argentina ni en la chilena ni, obviamente, en Tierra del Fuego.

A pesar de este aparentemente escaso resultado, existen pocos datos sobre la morfología, la biología y los estadios juveniles de la mayoría de estas especies y eran muy amplias las zonas de las que no existía dato alguno, hechos que contribuimos a mitigar con los datos que ahora aportamos.

\section{Chrysopidae Schneider}

Chrysopinae Schneider, 1851

Chrysopini Schneider, 1851

\section{Ungla argentina (Navás, 1911)}

Brooks \& Barnard, 1990: 276 (tax), González Olazo, 1996: 379 (list).

Hypocrhysa argentina Navás, 1911

Navás, 1911: 275 (des), 1913: 93 (morf, dis), Tjeder, 1966: 247 (tax), Adams, 1967: 221 (tax), Tjeder, 1971: 112 (tax), Adams, 1975: 169 (tax), Penny, 1977: 16 (list), Adams, 1978a: 292 (ref).

Chrysopa argentina (Navás, 1911)

Tjeder, 1966: 247 (tax), Stange, 1967: 31 (list), Tjeder, 1971: 113 (a, morf, dis, tax, ㅇ ), Adams, 1975: 169 (list), Penny, 1977: 16 (list), Brooks \& Barnard, 1990: 241 (ref).

Suarius argentinus (Navás, 1911)

Adams, 1975: 168 (tax), 169 (morf, a, ơ dis), 171 (nt), Adams \& Penny, 1986: 121 (morf, ㅇ, dis), 1987: 436 (ref).

= Ungla annulata Navás, 1914

Navás, 1914: 224 (des), Stange, 1967: 41 (list), Adams, 1975: 168 (list), 169 (tax), 171 (ref), Penny, 1977: 16 (list), Brooks \& Barnard, 1990: 276 (tax).

Material estudiado: 5: 24.X.01, 1 우 (SF), 8.XII.01, 1 우, 7.III.02, 1 우 (SF), 18.III.02, $10^{7}$ (SF), 22.III.02, 1 ㅇ․ . 6: 22.X.01, 1 ㅇ , 22.X.01, 1 ㅇ (SF), 22.XI.01, 1 ㅇ, 19.XII.01, $1 \sigma^{7}, 12$ 우, 1 우 (MACN), 28.II.02, $50^{7} \sigma^{7}, 10^{7}$ (MACN), 7 우 우 + 1 larva, 28.II.02, $10^{7}$ (SF). 7: 19.XII.01, $1 \sigma^{\top}$ (SF) + 3 larvas. 11: 9.II.02, $10^{7}, 2$ 우 우 .

Especie conocida de Argentina (Santiago del Estero, La Rioja, Catamarca y Tucumán) de biología casi desconocida.

Se han hallado numerosos individuos (29) sobre Jordina rhombifolia Hook et Arn., Santalaceae, con la que parece estar fuertemente asociada y sobre la que parece ser muy abundante, también se han recolectado ejemplares sobre algunas coníferas como Austrocedrus chilensis (Don.) Florin et Boutleje (3) y Pinus halepensis Mill. (1) y a la luz (7) y tres larvas sobre Acacia caven (Molina), Mimosaceae y una sobre Prosopis caldenia Bork., Mimosaceae. 
A

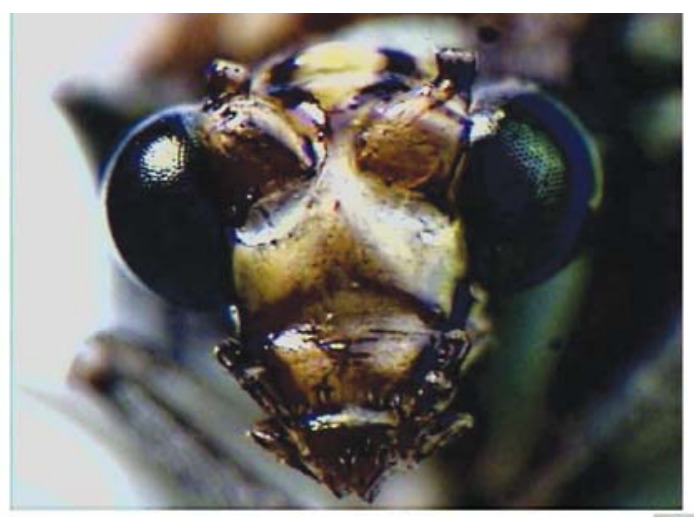

B
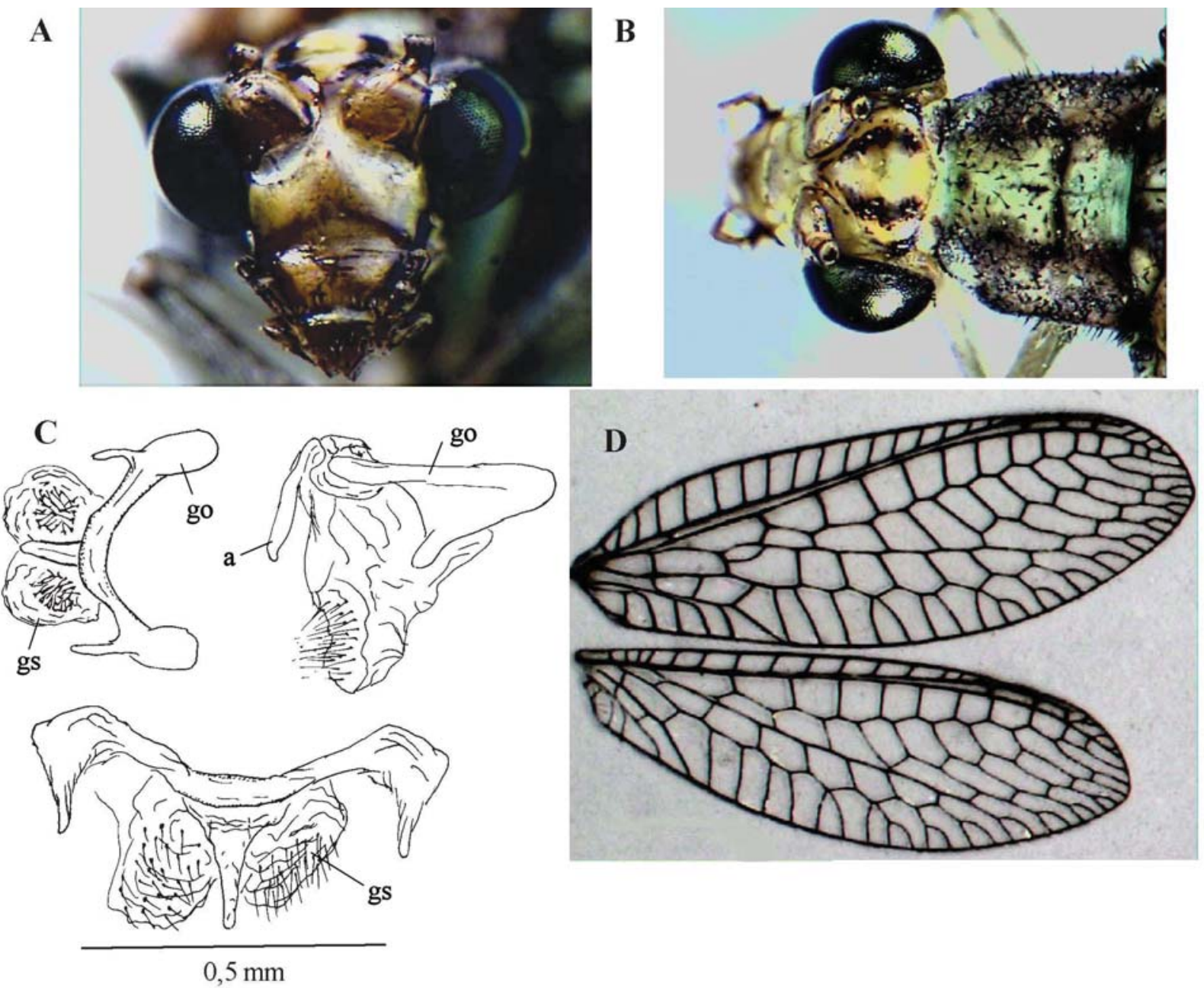

Fig. 1.-Ungla argentina (Navás, 1911). A: Cabeza, vista frontal. B: Cabeza y protórax, vista dorsal. C: Genitalia masculina interna, vista dorsal, lateral y frontal $\left(\mathrm{a}=\right.$ arceso, $\mathrm{go}_{\mathrm{o}}$ gonarco, $\mathrm{gs}=$ gonosaco $)$. D: Alas.

Fig. 1.-Ungla argentina (Navás, 1911). A: Head, frontal view. B: Head and prothorax, dorsal view. C: Internal male genitalia, dorsal, lateral and frontal views ( $\mathrm{a}=$ arcessus, $\mathrm{go}_{\mathrm{g}}=$ gonarcus, $\mathrm{gs}=$ gonosaccus). D: Wings.

Se anotan y describen de esta especie nuevos datos sobre su morfología y genitalia y se describen sus estadios preimaginales que eran desconocidos hasta la fecha.

Los imagos poseen la cabeza verde amarillenta (Figs. 1A, 1B) con manchas negras. Genas negras, vértex con dos estrías negras en forma de $\mathrm{V}$ y otra lateral cerca del ojo (Fig. 1B), sin mancha pos-ocular. Frente con una mancha negra en la parte central y una sobre el margen posterior del foramen, margen anterior del clípeo negro, labro pardo. Escapo amarillo con una mancha negra en su margen externo, pedicelo pardo, flagelo negro con filas trans- versales de sedas negras, escleritos laterales con una banda negra lateral. Palpos maxilares pardo oscuros, palpos labiales más pálidos. Tórax con pronoto, mesonoto y metanoto verde con manchas pardas irregulares y numerosas sedas negras y cortas, bandas laterales negras (Fig. 1B) encerrando una pequeña zona verde, meso y metaepímeros oscuros. Alas muy iridiscentes, las anteriores (Fig. 1D) de longitud: $1,38 \pm 0,12 \mathrm{~cm} /$ anchura $0,46 \pm$ $0,04 \mathrm{~cm}$. Venas transversales negras, venas costal y longitudinales verdes y oscuras en las intersecciones con las venas trasversales, célula intramediana con márgenes curvos, gradiformes internas (4-5) y 

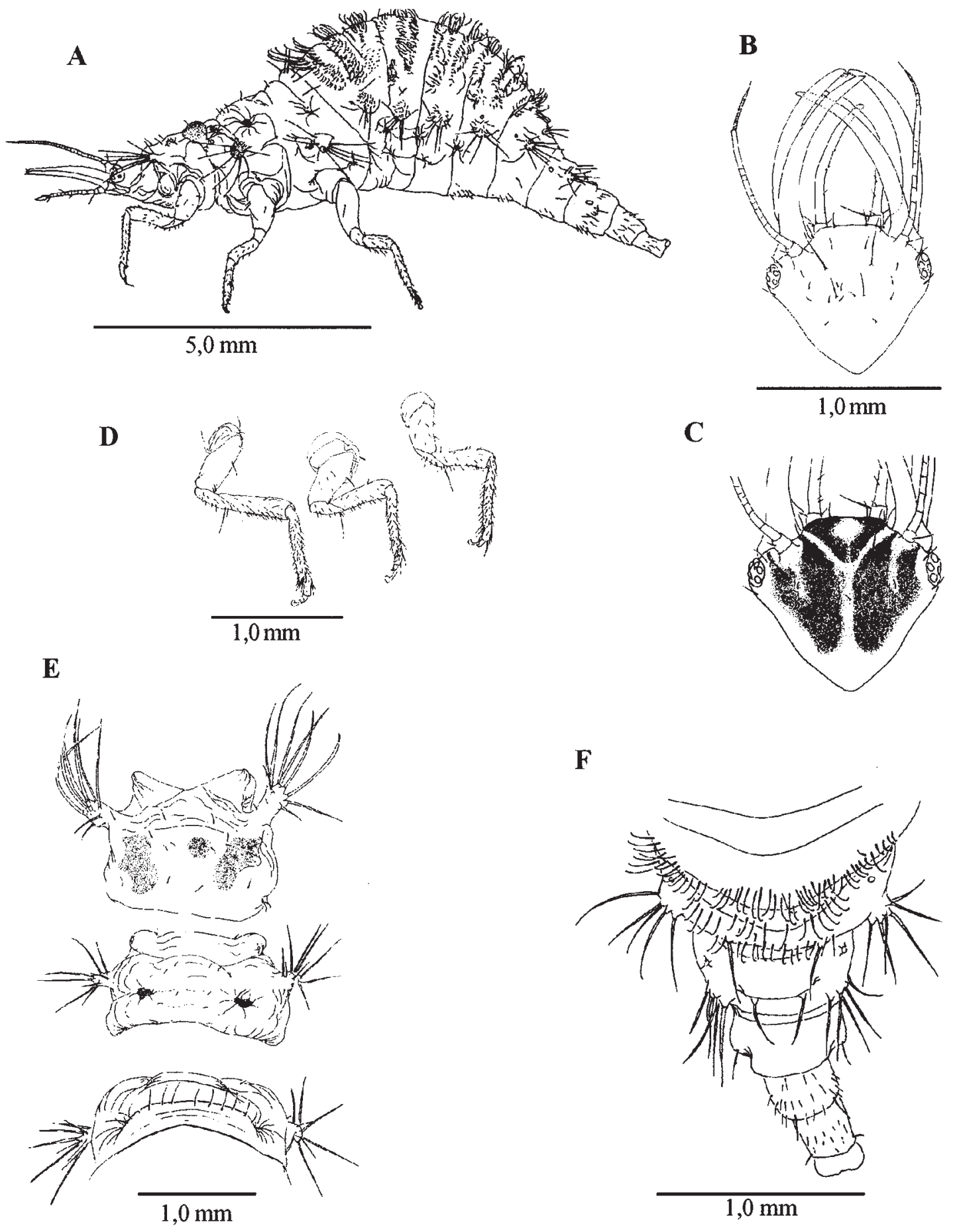

Fig. 2.- Larva desarrollada de Ungla argentina (Navás, 1911). A: Aspecto general, vista lateral. B: Cabeza, quetotaxia, vista dorsal. C: Cápsula cefálica, pigmentación, vista dorsal. D: Patas, vista lateral. E: Tórax, vista dorsal. F: Extremo abdominal, vista dorsal.

Fig. 2.- Fully developed larva of Ungla argentina (Navás, 1911). A: General, lateral view. B: Head, chaeatotaxy, dorsal view. C: Head capsule, pigmentation, dorsal view. D: Legs, lateral view. E: Thorax, dorsal view. F: Apex of the abdomen, dorsal view. 
extenas (5), pterostigma verde. Patas verdes con sedas negras y extremo basal de las tibias oscuro, uñas progresivamente dilatadas hacia su base. Abdomen verde con manchas negras en los terguitos. En el macho el esternito $8+9$ sin microtholi, gonarco estrecho y curvo caudalmente (Fig. 1C), rama externa del gonarco triangular con una proyección frontal digitiforme, arceso estrecho y largo, adelgazado apicalmente, gonosaco densamente cubierto de largas sedas (Fig. 1C).

Las puestas fueron obtenidas a partir de diferentes hembras que permanecieron vivas durante 7-9 días. Las puestas se depositaban en racimos colgantes en la tapa de la caja de cultivo en número de uno o dos racimos por hembra, cada uno de ellos con 8-10 huevos. Éstos son de color verde pálido y tornan a blancuzco primero y pardo después conforme progresa su desarrollo, y tardan entre 6 y 9 días en eclosionar. Las larvas neonatas permanecen varias horas sobre el corion antes de iniciar su actividad. Se les proporcionó ninfas e imagos de Aphis (Aphis) nerii Boyer de Fonscolombe, 1841, Macrosiphum (Macrosiphum) rosae rosae (Linnaeus, 1758) y Brachycaudus (Appelia) schwartzi (Börner, 1931) y son muy activas cubriéndose el dorso con restos de sus presas y otros elementos.

La larva de tercer estadio (Fig. 2) posee el cuerpo subcilíndrico y coloración pálida con manchas oscuras por toda su superfície. Superfície del tegumento dorsalmente revestido de microtriquias (Fig. 2A). Parte anterior del abdomen más voluminoso y arqueado dorsalmente (Fig. 2A). Dorso con alta densidad de setas típicas de larva portadora de restos. Tubérculos dorso-laterales digitiformes en el tórax (Fig. 2E) y levemente abultados en el abdomen (Fig. 2F). Posee tres tipos de sedas: lisa y recta, lisa en gancho y recta y espinosa. Cabeza pálida con manchas dorsales pardo oscuras en posición lateral y una mancha anterior según Fig. 2C. Las manchas laterales son más estrechas posteriormente y se bifurcan anteriormente envolviendo la base de las antenas (Fig. 2C), la mancha anterior es triangular con una parte central clara (Fig. 2C). Quetotaxia cefálica (Fig. 2B) con las sedas primarias $(\mathrm{C} 1-\mathrm{C} 12)$ presentes, sedas 1 y 11 largas, siendo la última mucho mayor. Mandíbulas de color ámbar, dentadas apicalmente, maxilas con 3 sedas apicales. Palpos labiales pardos pálidos, segmento basal con una larga seda y un par de sedas cortas, los demás segmentos con sedas laterales. Antena pálida, anillada, flagelo con una larga seda apical y escapo con una larga seda externa (Fig. 2B).

Tórax (Fig. 2E) achatado con tubérculos laterales digitiformes, siendo el primer par mucho más largo que el segundo y éste más largo que el tercero, sedas dispersas distribuídas sobre el dorso (Fig. 2E). Protórax con proyecciones laterales con 10 sedas apicales aserradas y espinosas, dirigidas frontalmente, porta tres escleritos oscuros, dos mayores dorso-laterales y uno menor central (Fig. 2E) y varias sedas pequeñas sobre el dorso. Mesotórax con proyecciones laterales cortas y 9 largas sedas aserradas y espinosas, porta dos pequeños escleritos oscuros laterales situados dentro de una pequeña cavidad y porta pequeñas sedas sobre el dorso (Fig. 2E). Metatórax con pequeñas proyecciones laterales con 8 sedas largas, aserradas y espinosas y porta una línea transversal con 12 sedas ganchudas (Fig. 2E). Patas (Fig. 2D) de color ámbar claro con pequeñas manchas, procoxa, mesocoxa y metacoxa ámbar portadoras de una larga seda, pro y mesocoxas con sedas dispersas, trocánteres con una larga seda distal a las otras sedas cortas, fémures y tibias densamente cubiertos de sedas negras, tarsómero proximal con largas sedas, uñas levemente curvadas y empodio oscuro.

Abdomen dos veces más voluminoso que el tórax (Fig. 2A), dorso cubierto de filas trasversales de setas en gancho, siendo más largas en los dos primeros segmentos. Tubéculos laterales con largas setas apicales, tubérculos dorsales con setas largas sobre los segmentos 6 y 7 (Fig. 2F).

Ungla binaria (Navás, 1922)

Brooks \& Barnard, 1990: 240 (tax, morf, a, ơ, 우), 276 (tax).

Chrysopa binaria Navás, 1922 nec 1928

Navás, 1922a: 191 (des), 1926: 108 (dis), Stange, 1967: 31

(list), Penny, 1977: 16 (list), González Olazo, 1996: 378 (list).

Material estudiado: 5: 17.II.02, 2 우 우 a la luz (SF). 6: 28.II.02, $10^{7}, 2$ 우 우 sobre Jordina rhombifolia Hook et Arn., Santalaceae, 28.II.02, $10^{7}$ (SF) sobre Jordina rhombifolia. 7: 30.XII.01, 1 ㅇ sobre Pinus halepensis.

Especie sólo conocida de Argentina (Córdoba) y de biología prácticamente desconocida.

Se anotan y describen de esta especie nuevos datos sobre su morfología, su genitalia y sus estadios juveniles que eran desconocidos hasta la fecha.

El imago de esta especie es semejante al de Ungla argentina, pudiendo diferenciarse a través de las siguientes características: Cabeza con frente blanca sin manchas, clípeo y labro de color ámbar sin manchas (Figs. 3A, 3B), mancha pos-ocular parda, escapo con mancha lateral apical y mancha parda extendiéndose hasta la fosa antenal membranosa (Fig. 3A). Alas anteriores (Fig. 3D) de longitud: $1,1 \mathrm{~cm} /$ anchura $0,4 \mathrm{~cm}$. Genitalia del macho 
A

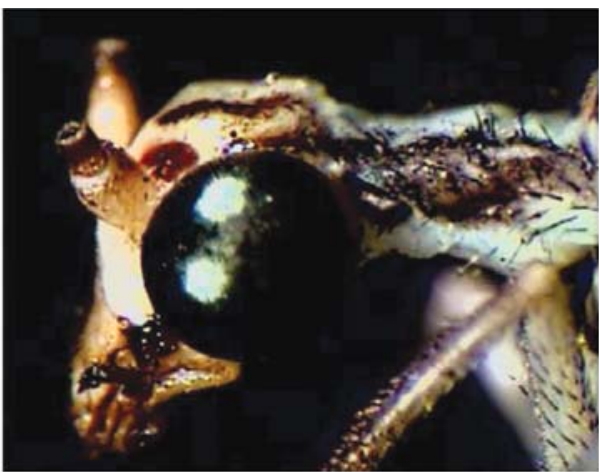

C

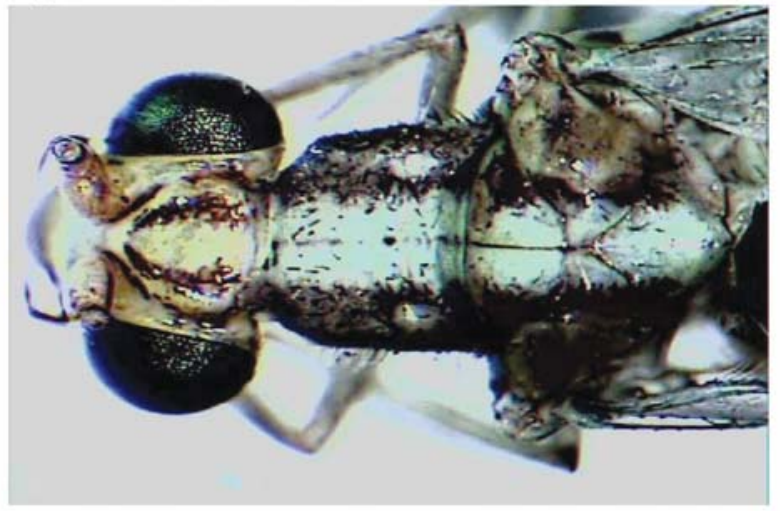

B

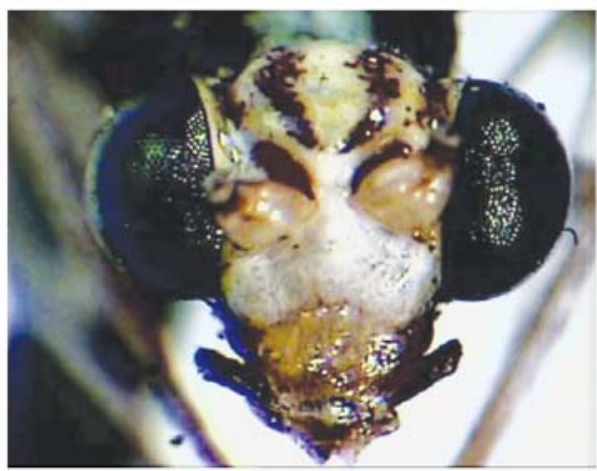

D

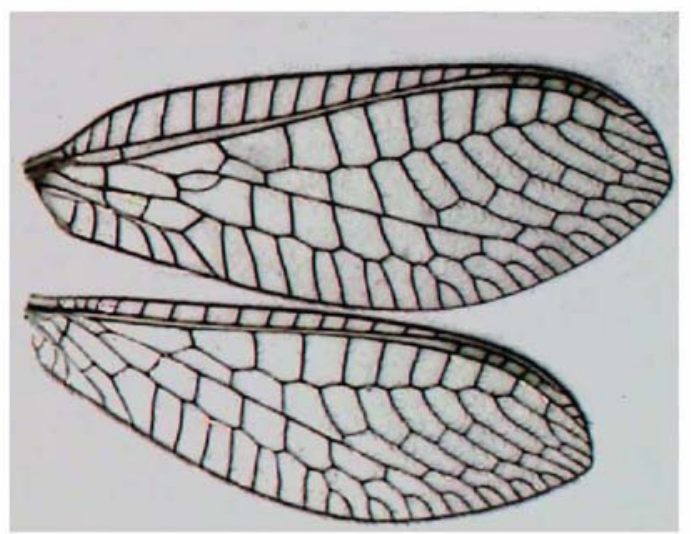

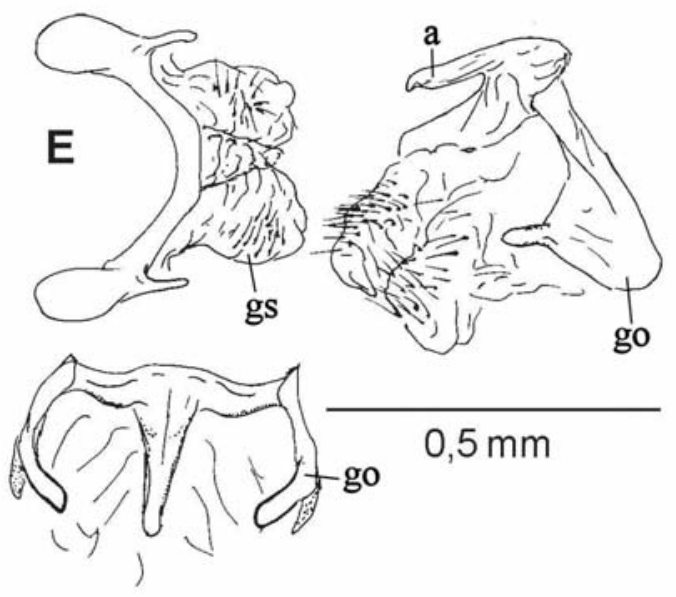

$\mathbf{F}$

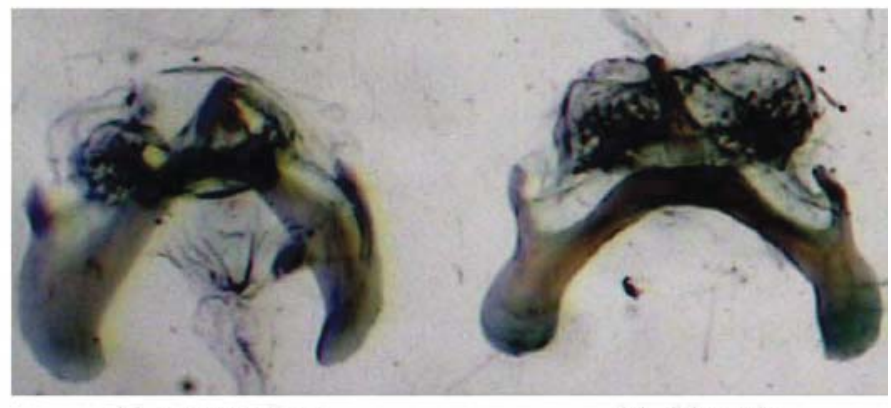

U. argentina

U. binaria

Fig. 3.- Ungla binaria (Navás, 1922). A: Cabeza y protórax, vista lateral. B: Cabeza, vista frontal. C: Cabeza, protoráx y mesotórax, vista dorsal. D: Alas. E: Genitalia masculina interna, vista dorsal, lateral y frontal $(\mathrm{a}=\mathrm{arceso}, \mathrm{go}=\mathrm{gonarco}, \mathrm{gs}=\mathrm{gonosaco})$. F: Genitalia interna de Ungla argentina y U. binaria, vista dorsal.

Fig. 3.- Ungla binaria (Navás, 1922). A: Head and prothorax, lateral view. B: Head, frontal view. C: Head, pro- and mesothorax, dorsal view. D: Wings. E: Internal male genitalia, dorsal, lateral and frontal views (a= arcessus, go= gonarcus, gs= gonosaccus). F: Internal genitalia of Ungla argenitina and U. binaria, dorsal view. 


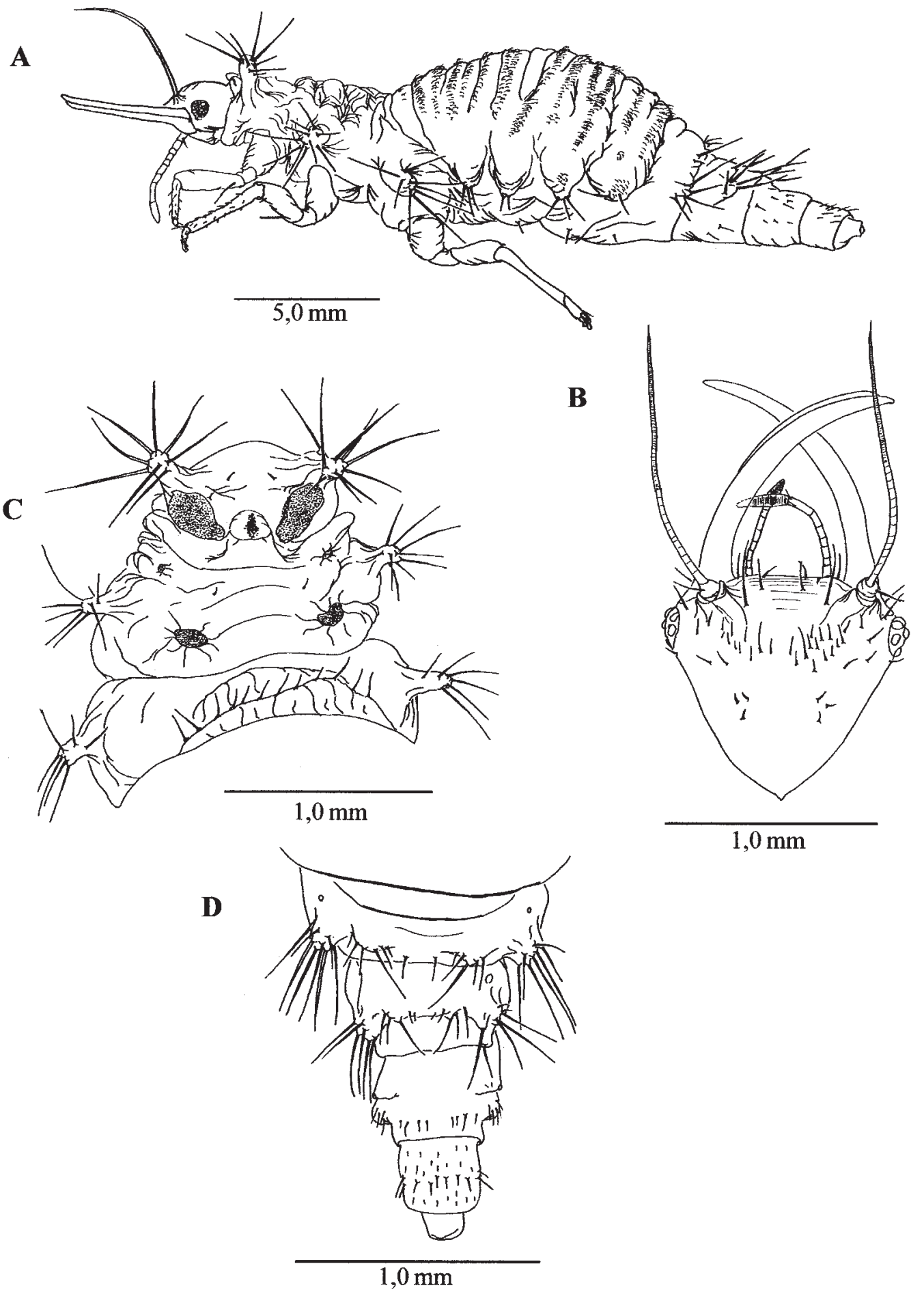

Fig. 4.- Larva desarrollada de Ungla binaria (Navás, 1922). A: Aspecto general, vista lateral. B: Cabeza, quetotaxia, vista dorsal. C: Tórax, vista dorsal. D: Extremo abdominal, vista dorsal.

Fig. 4.- Fully developed larva of Ungla binaria (Navás, 1922). A: General, lateral view. B: Head, chaetotaxy, dorsal view. C: Thorax, dorsal view. D: Apex of the abdomen, dorsal view. 
con gonarcus más estrecho y largo, proceso digitiforme de la rama del gonarco más delgado y largo, arceso más largo y grueso (Figs. 1C, 3E, 3F).

Las puestas fueron obtenidas a partir de la última hembra citada que permaneció viva durante 10 días. Las puestas se depositaban en racimos colgantes en la tapa de la caja de cultivo en número de dos racimos, cada uno de ellos con 9-10 huevos. Éstos son de color verde pálido y tornan a blancuzco primero y pardo después conforme progresa su desarrollo, y tardan entre 8 y 9 días en eclosionar. Las larvas neonatas permanecen varias horas sobre el corion antes de iniciar su actividad. Se les proporcionó ninfas e imagos de Aphis (Aphis) nerii, Macrosiphum (Macrosiphum) rosae rosae y Brachycaudus (Appelia) schwartzi y son muy activas cubriéndose el dorso con restos de sus presas y otros elementos.

La larva desarrollada (Fig. 4) es semejante a la de Ungla argentina (Fig. 2). Se diferencia por la quetotaxia cefálica por su mayor densidad de setas, tubérculos torácicos laterales más largos, tubérculos laterales y medianos de los segmentos abdominales 6 y 7 mayores y con setas más largas y setas dorsales en gancho menores (Figs. 2, 4).

Chrysopodes (Neosuarius) porterina (Navás, 1910) Adams \& Penny, 1987: 436 (tax), Brooks \& Barnard, 1990: 240 (a), 272 (list).

Chrysopa porterina Navás, 1910

Navás, 1910a: 70 (des), 1910b: 47 (des), 1910c: 238 (morf, dis), 1913: 89 (morf, dis), 1925a: 305 (morf, dis), 1929a: 19 (dis), Penny, 1977: 20 (list).

Chrysopa porteri Navás, 1910 (lapsus)

Navás, 1928: 98 (dis), Penny, 1977: 20 (list).

Material estudiado: 1: 21.X.01, 1 우 sobre Balbisia peduncularis, D. Don., Cistaceae. 2: 18.X.01, $10^{\text {T }}$ sobre Bahia ambrosioides Lag., Cistaceae (SF). 13: 4.II.02, 1 ㅇ sobre Austrocedrus chilensis.

Especie muy poco conocida. Citada de Chile (Valparaíso, Maule, Nos, La Serena). Se dan los primeros datos sobre su biología y se cita por primera vez para Argentina.

Se anotan y describen de esta especie nuevos datos sobre su morfología y su genitalia y a partir de estos ejemplares se obtuvieron puestas que nos permiten describir sus estadios juveniles que eran desconocidos hasta la fecha.

En el imago la cabeza (Fig. 5A) es pardo amarillenta con manchas negras. Gena negra, vértice con manchas negras y mancha pos-ocular presente. Frente con mancha negra sobre el margen lateral, clípeo negro y labro negro. Escapo y pedicelo ante- nal amarillo con manchas negras, fosa antenal negra, flagelo negro con filas transversales de setas negras. Escleritos cervicales con manchas negras lateralmente.

Tórax con pronoto, mesonoto y metanoto amarillos con muchas setas negras cortas, bandas laterales negras (Fig. 5C). Meso y meta epímeros oscuros. Alas anteriores (Fig. 5B) con longitud: $1,28 \mathrm{~cm} /$ anchura $0,4 \mathrm{~cm}$, venas transversales negras, vena costal y longitudinales negras, célula intramediana con márgenes curvos, gradiformes internas (4-5) y externas (7), pterostigma verde. Patas verdes con numerosas setas negras.

Abdomen negro, en el macho el esternito $8+9$ sin microtholi, gonarco estrecho y recto (Fig. 5E), rama lateral del gonarco triangular, arceso ancho en forma de quilla y punteagudo apicalmente (Fig. 5E), esternito $8+9$ punteagudo con numerosas sedas largas y negras. Ápice del ectoprocto terminando en un proceso puntiagudo (Fig. 6D).

Las puestas estaban formadas por 8 y 10 huevos que fueron depositados aisladamente y mayoritariamente pendiendo de la tapa de la caja que las contenía. Son verde pálidos y de pedúnculo proporcionalmente corto, y tornan a pardo conforme su desarrollo progresa. Su desarrollo embrionario duró entre 7-9 días. Las larvas mostraron un marcado comportamiento caníbal. Se cubren con abundantes restos de presas, sus propias exuvias y otros elementos de forma exagerada o desproporcionada para su tamaño en relación a otros géneros de crisopas cuyas larvas practican este comportamiento defensivo, y se balancean llamativamente al caminar.

Algunas de ellas fueron alimentadas con ninfas e imagos de áfidos Macrosiphum (Macrosiphum) rosae rosae y puparon a los 23 días de su nacimiento, otras lo fueron inicialmente con Brachycaudus (Appelia) schwartzi deteniendo su actividad y desarrollo a partir de la primera muda o muriendo, por lo que posteriormente se les ofreció de nuevo Macrosiphum (Macrosiphum) rosae rosae sin mejores resultados y a la vista del tipo de planta (conífera) sobre la que fue hallado su progenitora, se les proporcionó otro tipo de áfido, en este caso de conífera (Pinus halepensis): Eulachnus tauricus Bozhko, 1961. De ellas algunas retomaron aparentemente su actividad normal e iniciaron su pupación a los 34 días de su nacimiento y otras detuvieron de nuevo su actividad manteniéndose inactivas pero vivas hasta 65 días después de su nacimiento y algún ejemplar en fase de pupa murió al emerger como adulto. De todo ello se deduce un irregular desarrollo preimaginal 


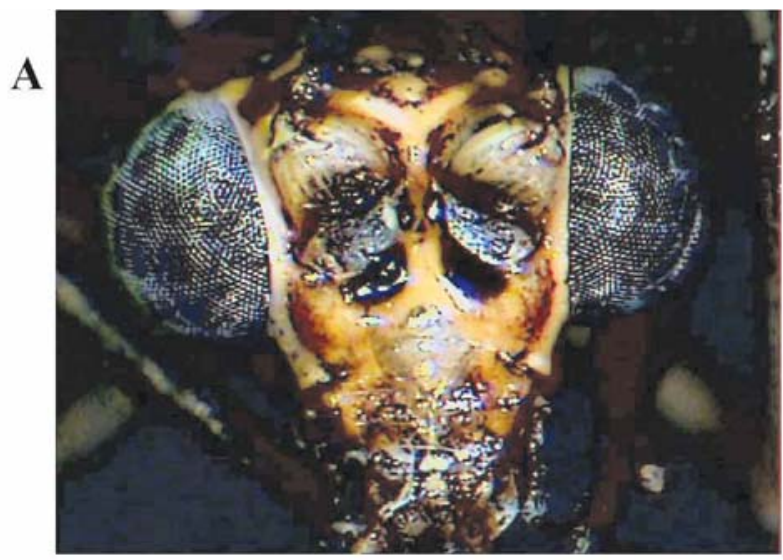

B
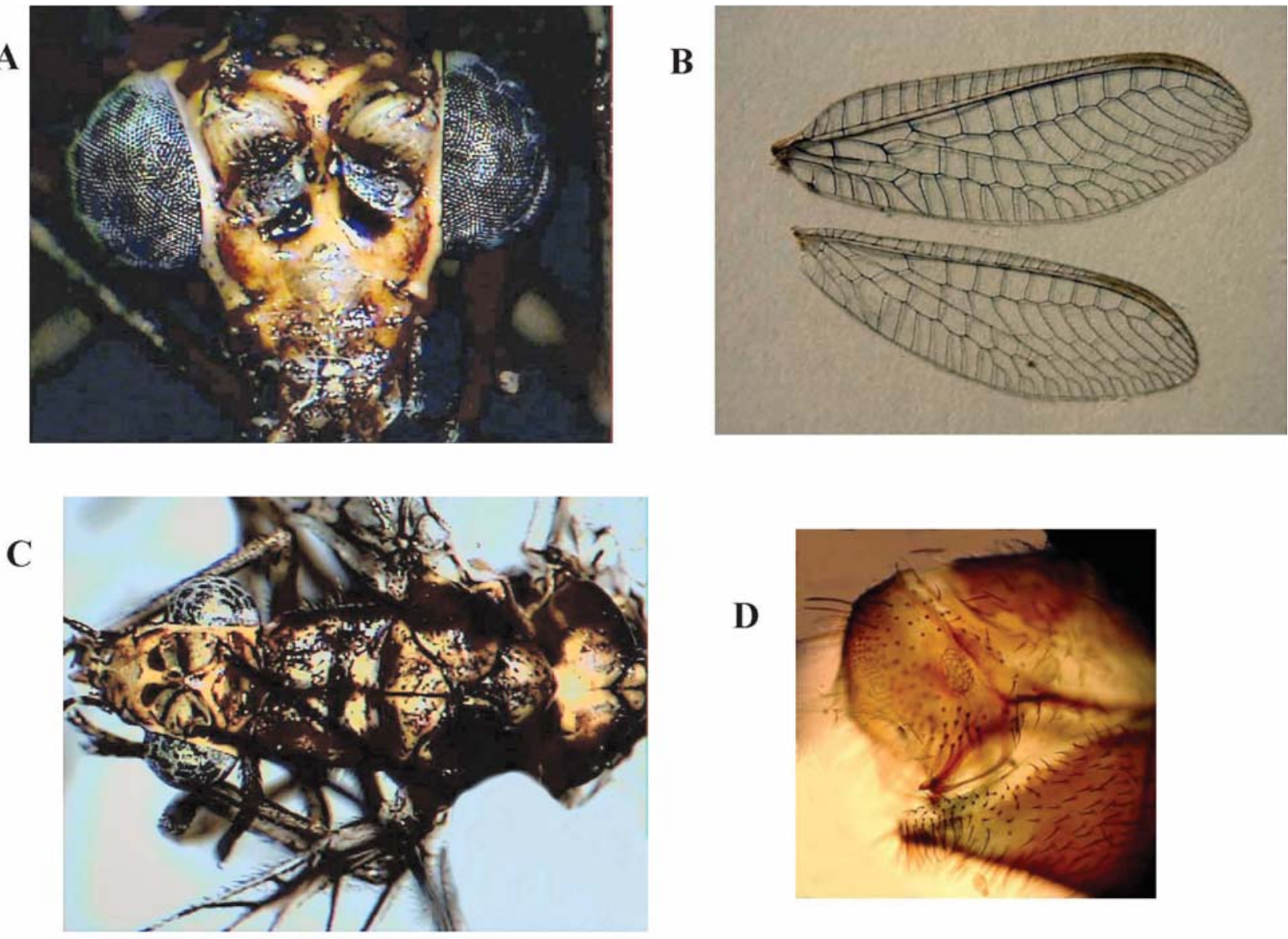

\section{D}

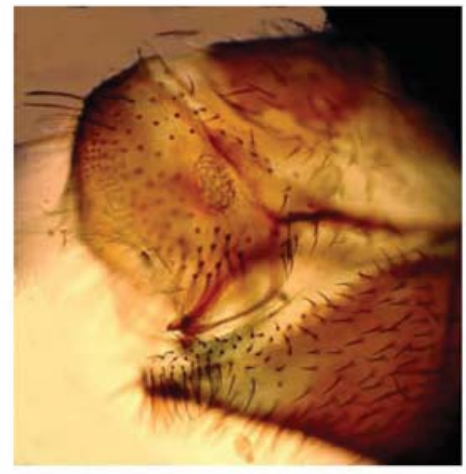

E

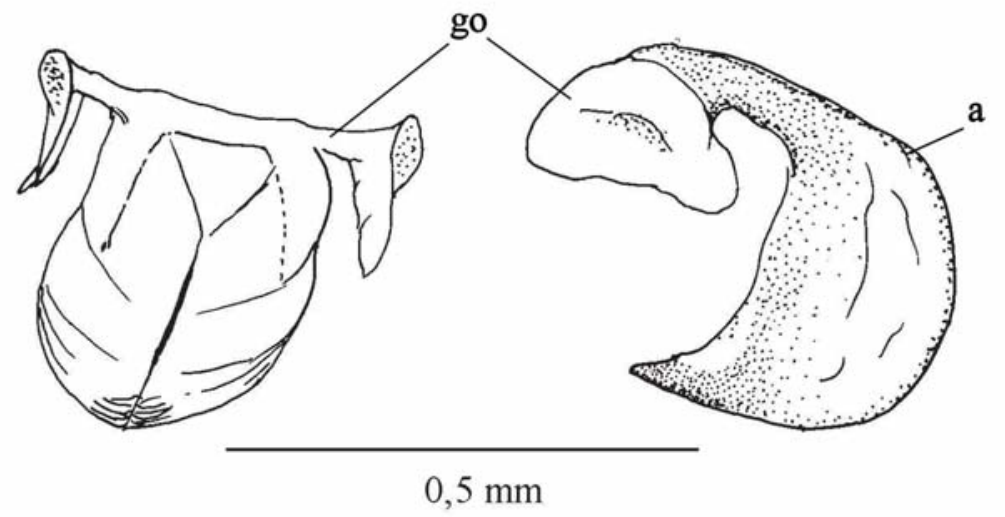

Fig. 5.- Chrysopodes (Neosuarius) porterina (Navás, 1910). A: Cabeza, vista frontal. B: Alas. C: Cabeza, protórax, mesotórax y metatórax, vista dorsal. D: Macho, ápice del abdomen, vista lateral. E: Macho, genitalia interna, vista dorsal y lateral (a= arceso, $\mathrm{go}=$ gonarco).

Fig. 5.-Chrysopodes (Neosuarius) porterina (Navás, 1910). A: Head, frontal view. B: Wings. C: Head, pro-, meso- and metathorax, dorsal view. D: Male, apex of the abdomen, lateral view. E: Male, internal genitalia, dorsal and lateral view $(a=a r c e s-$ sus, go= gonarcus). 
seguramente derivado de una aparente y marcada especificidad alimentaria.

Las larvas neonatas son pálidas con una débil línea media parda y una a cada lado más ancha. En fases más avanzadas poseen setas negras y manchas negras en la cabeza y el tegumento del tórax es rosáceo con dos tenues bandas pardas dorsales, una a cada lado y dos bandas longitudinales negras sobre la región ventral de los segmentos del abdomen.

Las larvas desarrolladas (Fig. 6) poseen el cuerpo cilíndrico y la superficie del tegumento dorsalmente revestido de bandas de microtriquias y setas típicas de larva portadora de restos. Parte anterior del abdomen más voluminoso y muy arqueado dorsalmente (Fig. 6A).

Cabeza (Fig. 6B) pálida con dos pares de estrías oscuras obliquas, las laterales más estrechas y largas que las centrales. Quetotaxia cefálica (Fig. 6B) con las setas primarias $(\mathrm{C} 1-\mathrm{C} 12)$ presentes, setas 1 y 11 largas siendo la última mucho mayor. Mandíbulas ámbar, dentadas apicalmente, maxilas con setas apicales. Palpos labiales pardo claro, segmento basal con una larga seda y un par de sedas cortas, demás segmentos con setas laterales. Antena pálida, anillada, flagelo con seta apical larga, escapo con una seta larga.

Tórax (Figs. 6B, 6D, 6E, 6F) aplanado con tubérculos laterales digitiformes siendo el primer par mucho más largo que el segundo y éste más largo que el tercero, setas distribuídas en el dorso de dos tipos: lisa en gancho y recta espinosa. Protórax con proyecciones laterales largas con 10 setas apicales (aserradas y espinosas) dirigidas frontalmente, porta varias setas pequeñas y un par de setas largas en el dorso. Mesotórax con proyecciones laterales cortas con 9 setas (aserradas y espinosas) y porta pequeñas setas distribuídas sobre el dorso. Metatórax con pequeñas proyecciones laterales con 8 setas largas (aserradas y espinosas) y posee una línea transversal en posición dorsomedial con 12 setas en gancho (Fig. 6F). Patas (Fig. 6C) de color ámbar claro, pro, meso y metacoxa ámbar, con una larga seta, pro y mesocoxa con setas dispersas, trocánteres con una seta larga distal a las otras setas cortas, fémures y tibias densamente cubiertos de setas negras, tarsómero proximal con largas setas y uñas levemente curvadas con empodio oscuro.

Abdomen más voluminoso que el tórax, dorsalmente cubierto de filas trasversales de setas en gancho, siendo las de los dos primeros segmentos más largas, tubérculos laterales con largas setas apicales, tubérculos dorsales con setas largas sobre el dorso de los segmentos 6 y 7 (Fig. 6G).

\section{Chrysoperla externa externa (Hagen, 1861)}

Adams, 1983: 44 (morf, tax, ơ, dis), Brooks \& Barnard, 1990: 205 (ref, biol), 271 (tax, list).

Chrysopa externa Hagen, 1861 nec Timberlake, 1920

Hagen, 1861: 221 (des), Navás, 1922a: 190 (dis), Adams, 1962: 222 (ref), Tauber, 1974: 1151 (dis, la), Adams \& Penny, 1987: 420 (tax, morf, a, ơ, ㅇ, dis), González Olazo, 1987: 41 (dis, bio), Brooks, 1994: 168 (tax, morf, a, ơ , 우, dis).

Chrysoperla externa (Hagen, 1861)

Adams \& Penny, 1986: 120 (morf, ㅇ, dis), 1987: 420 (tax), Carvalho et al., 1998: 83 (biol, dis, la), Auad et al., 2001a: 455 (dis, la, biol), Auad et al., 2001b: 429 (dis, la, biol), Freitas \& Penny, 2001b: 247 (la), 268 (morf, a, o", 우, dis, biol), Penny, 2002: 219 (morf, a, dis, biol), Figueira et al., 2002: 133 (dis, biol), Auad et al., 2002: 536 (dis, la, biol).

= Chrysopa plorabunda Fitch, 1856

Muma, 1959: 26 (morf, dis, la) sensu Tauber, 1974.

$=$ Chrysopa lanata $($ Banks, 1910)

Banks, 1910: 154 (des), Navás, 1914: 221 (dis), Bruch, 1917: 361 (morf, dis, h, la, pp, a, biol), Navás, 1917a: 195 (dis), 1919a: 300 (dis), 1920: 49, 59 (dis), $1921 \mathrm{a:} 259$ (dis), 1921b: 51 (dis), 1922a: 190 (dis), 1922b: 361 (dis), 1922c: 23 (dis), Esben Petersen, 1924: 310 (a, dis), Navás, 1925b: 20 (dis), 1926: 108 (dis), 1927a: 324 (dis), 1929b: 222 (dis), 1929c: 357 (dis), 1930: 64 (dis), Williams, 1931: 130 (bio, la), Navás, 1932a: 20 (dis), 1933: 305 (dis), Zimmerman, 1957: 90 (dis, biol, a, o'), Adams, 1962: 222 (dis, ơ), Stange, 1967: 34 (list), Ru et al., 1975: 187 (h, la, biol, dis), Penny, 1977: 19 (list), Crouzel \& Saini, 1979 (dis, biol, la), Adams \& Penny, 1987: 421 (tax), Brooks \& Barnard, 1990: 271 (tax, list), Brooks, 1994: 168 (tax).

$=$ Chrysopa lanata lanata (Banks, 1910)

Stange, 1967: 34 (list), Crouzel \& Botto, 1976: 2 (morf, dis, la, biol), Botto \& Crouzel, 1979: 745 (dis, biol).

= Chrysopa lanata var. basalis Navás, 1920

Navás, 1920: 59 (des), Navás, 1922a: 190 (dis), 1927a: 324 (dis), Stange, 1967: 34 (list), Penny, 1977: 19 (list), Adams \& Penny, 1987: 421 (tax), González Olazo, 1996: 379 (list).

= Chrysopa lanata var. climacia Navás, 1917

Navás, 1917b: 495 (des), Navás, 1922a: 190 (dis, tax), 1923: 116 (dis, tax), Stange, 1967: 34 (list), Penny, 1977: 19 (list), Adams \& Penny, 1987: 421 (tax), González Olazo, 1996: 379 (list).

= Chrysopa lanata var. platensis Navás, 1915 Navás, 1915: 132 (des), Stange, 1967: 35 (list), Penny, 1977: 19 (list), Adams \& Penny, 1987: 421 (tax), González Olazo, 1996: 379 (list).

= Chrysopa lanata var. unita Navás, 1919

Navás, 1919b: 24 (des), Navás, 1920: 49 (dis), Stange, 1967: 35 (list), Penny, 1977: 19 (list), Adams \& Penny, 1987: 421 (tax).

= Chrysopa lanata var. gradata Navás, 1919

Navás, 1919a: 300 (des), Stange, 1967: 34 (list), Penny, 1977: 19 (list), Adams \& Penny, 1987: 421 (tax), González Olazo, 1996: 379 (list).

= Chrysopa lanata var. picta Navás, 1932 Navás, 1932b: 80 (des), Stange, 1967: 35 (list), Penny, 1977: 19 (list) 


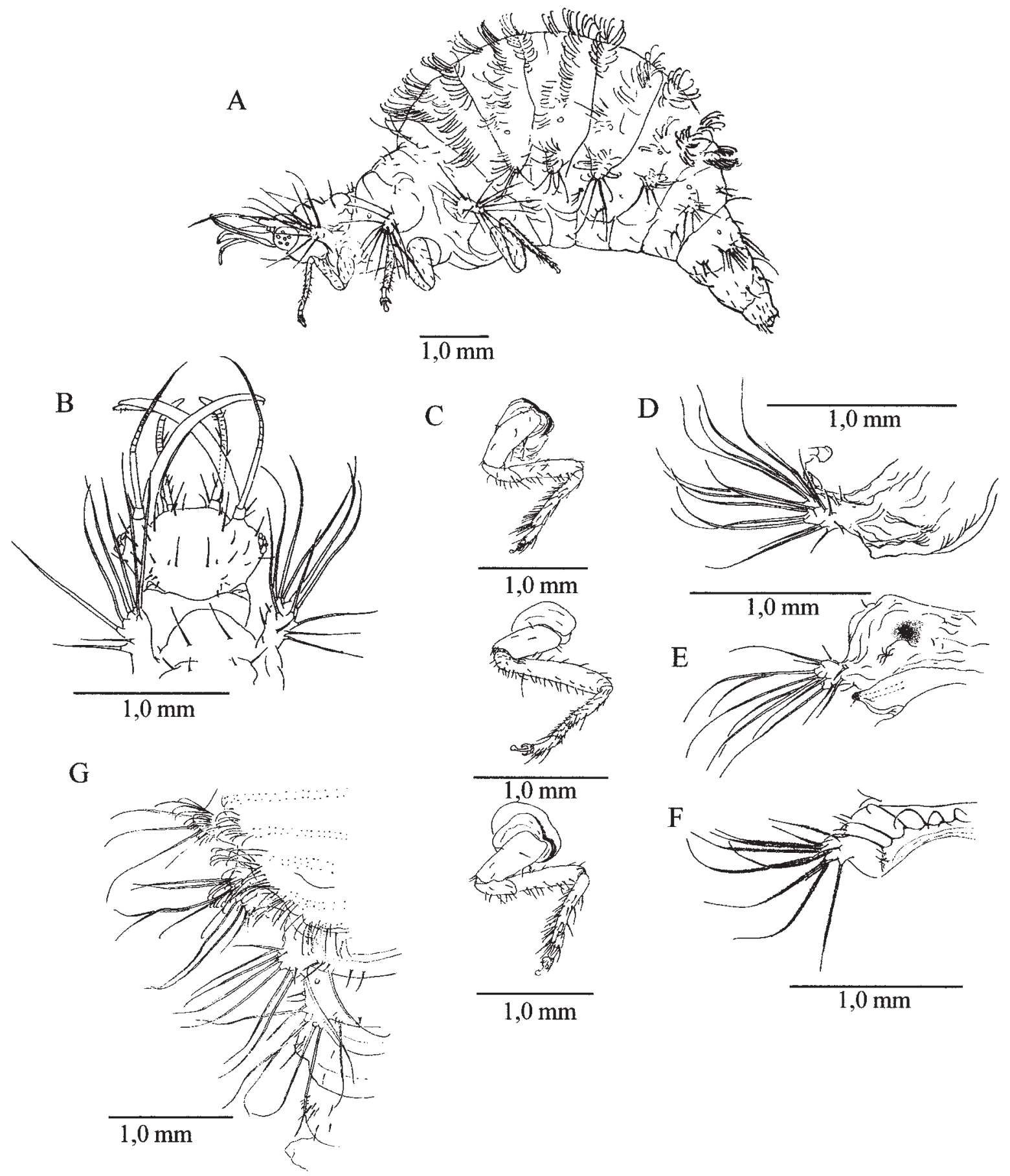

Fig. 6.- Larva desarrollada de Chrysopodes (Neosuarius) porterina (Navás, 1910). A: Aspecto general, vista lateral. B: Cabeza y pronoto, quetotaxia, vista dorsal. C: Patas, vista lateral. D: Porción lateral del pronoto, vista dorsal. E: Porción lateral del mesonoto, vista dorsal. F: Porción lateral del metanoto, vista dorsal. G: Porción lateral del extremo abdominal, vista dorsal.

Fig. 6.- Fully developed larva of Chrysopodes (Neosuarius) porterina (Navás, 1910). A: General, lateral view. B: Head and pronoto, chaetotaxy, dorsal view. C: Legs, lateral view. D: Lateral portion of the pronoto, dorsal view. E: Lateral portion of the mesonoto, dorsal view. F: Lateral portion of the metanoto, dorsal view. G: Lateral portion of the apex of the abdomen, dorsal view. 
= Chrysopa graciana Navás, 1919

Navás, 1919a: 301 (des), Navás, 1922a: 190 (dis), 1927b: 21 (dis), 1929b: 222 (dis), Stange, 1967: 33 (list), Adams, 1975: 169 (tax), Penny, 1977: 16 (list), Adams \& Penny, 1987: 421 (tax), Brooks, 1994: 168 (tax), González Olazo, 1996: 379 (list).

Chrysoperla gracina (Navás, 1919) (lapsus)

Brooks \& Barnard, 1990: 271 (tax, list).

Material estudiado: $3:$ 15.XI.01, 1 . 4: 18.XII.01, $1 \sigma^{7}$ (MACN). 6: 19.XII.01, 10", 2 우 우 (SF). 7: 3.XII.01, $2 \sigma^{\circ} \sigma^{\prime \prime}$, 1 우, 19.XII.01, $20^{7} \sigma^{7}, 1$ 우, $20^{\pi} \sigma^{7}$ (SF). 8: 8.XII.01, 2 우 우, 8.XII.01, $2 \sigma^{7} \sigma^{\pi}$ (SF). 9: 13.XII.01, 1 ㅇ․ 10: 3.XII.01, 1 ㅇ․ 12 : 3.XII.01, 1 우 .

Especie ampliamente citada desde el sur de EEUU, México y Centroamérica (Guatemala, Honduras, Costa Rica, Belice) y Caribe (Cuba, Puerto Rico, Haití, Bermudas, Bahamas, Barbados, Caimán, Trinidad) a Sudamérica (Brasil, Paraguay, Bolivia, Colombia, Venezuela, Perú, Ecuador, Juan Fernández, Chile, Argentina, Uruguay), con varias subespecies y variedades descritas de Argentina, Colombia, Costa Rica, Guatemala y Cuba.

En la zona de estudio se ha citado en Chile de Arica y en Argentina de Córdoba, Mendoza, Delta del Paraná, Buenos Aires, La Plata, Misiones, Tucumán, Jujuy, Santa Fe, Alta Gracia, Salta, Catamarca, Santiago del Estero y Río Negro.

Se trata de una especie muy común y eurioica, citada sobre muy diversos sustratos vegetales arbóreos y herbáceos (Freitas \& Penny, 2001b), nosotros la hemos hallado sobre Pinus halepensis (7 ejemplares), Jordina rhombifolia (6), Tamarix gallica L., Tamaricaceae (3), Acacia caven (2), Crataegus monogyna Jacq., Rosaceae (1) y Prosopis caldenia (1).

Como ocurre con Chrysoperla carnea (Stephens, 1836) del Paleártico Occidental, es una especie muy utilizada recientemente en la Región Neotropical para multitud de estudios experimentales y de laboratorio sobre control biológico, resistencia a insecticidas, dinámica de poblaciones, efectos de temperatura, fotoperíodo, diapausa, etc., que no hemos incluido en sus referencias faunísticas, morfológicas o taxonómicas anteriormente listadas.

\section{Chrysoperla defreitasi Brooks, 1994}

Brooks, 1994: 166 (des), Freitas \& Penny, 2001b: 268 (morf, a, ơ, dis, biol), Freitas, 2003: 385 (ref), 387 (morf, cl).

MATERIAL ESTUDiado: 5: 17.XI.01, $10^{7}, 17 . I I .02,1$ ㅇ a la luz. 7: 19.XII.01, $10^{7}$ sobre Jordina rombifolia (SF).

Especie sólo conocida de Brasil (Jaquaritingas, Sao Paulo, Mato Grosso) que se cita por primera vez en Argentina.
La hembra citada puso 12 huevos verdes y aislados durante la noche. Las neonatas nacen a los tres días y no prosperaron tras ofrecerles ninfas de Brachycaudus (Appelia) schwartzi.

\section{Conclusiones}

A pesar del esfuerzo realizado tras visitar 6 localidades de Coquimbo (Chile) y 53 localidades de Patagonia y Tierra del Fuego (Argentina y Chile) en las que se realizó la toma de muestras (Monserrat, 2003, 2005), sólo se han encontrado 73 ejemplares pertenecientes a 5 especies de crisópidos en sólo 13 de estas localidades ( 2 de Coquimbo en Chile y 11 de la Patagonia Argentina), todas situadas sobre el límite norte de la Patagonia y siempre por encima del paralelo $41^{\circ} 50$ 'S , a diferencia de otras familias de neurópteros como Coniopterygidae y especialmente Hemerobiidae (Monserrat, 2003, 2005) que llegan en su distribución a Tierra del Fuego y alcanzan el límite más meridional del Continente Americano, y no hemos recolectado ejemplares de esta familia en localidades más meridionales al paralelo citado.

Aunque muchas de las citas argentinas y chilenas de crisópidos existentes en la bibliografía son poco precisas e inducen a ciertas dudas debido a la homonimia en algunas de las toponimias y localidades citadas, la mayoría de los datos existentes se sitúan por encima del paralelo $36^{\circ} \mathrm{S}$ y todas parecen situarse por encima del paralelo $42^{\circ} \mathrm{S}$ (la cita más meridional de estas especies está referida por Navás, 1917a en Río Negro, sin más datos).

Debido a las condiciones climatológicas tan particulares en las zonas de la Región Neotropical muestreadas, marcadamente xéricas en Coquimbo y especialmente severas en la extensa zona meridional de Patagonia y Tierra del Fuego, sobre todo por la presencia de fuertes y persistentes vientos, la fuerte radiación solar, la existencia de un relativamente corto periodo de bonanza primaveral-estival con duras condiciones invernales y la deforestación que ha generado pobreza y uniformidad en sus medios -sólo más diversificados en las zonas montanas de influencia andina y pacífica- cabría esperar pocos resultados tras unos muestreos en los que se trataba de localizar un grupo tan termófilo y tan sensible al viento, especialmente por el tipo de muestreo mediante mangueo de la vegetación anteriormente citado. Parece evidente que estas condiciones ambientales limitan fuertemente la distribución general de esta familia en el Cono Sur Americano. No se ha hallado ningún ejemplar por 
encima de $655 \mathrm{~m}$, y sin embargo han sido hallados en localidades situadas entre -24 y $-45 \mathrm{~m}$ bajo el nivel del mar, hecho poco habitual.

En cualquier caso, existían muy pocos datos sobre la morfología y la biología de la mayoría de las especies de esta región, de la mayoría se desconocían sus estadios juveniles y eran muy amplias las zonas en las que no se había muestreado y de las que no existía dato alguno. Contribuimos a mitigar la falta de conocimiento sobre la distribución general de la familia en estas zonas y aportamos nuevos datos sobre su biología, morfología y estadios larvarios. Los datos aportados permiten presumir mayoritariamente poblaciones relativamente bajas en número de ejemplares y bastante generalistas en su elección de sustrato vegetal. Sin embargo otras especies parecen muy localizadas en un determinado medio y en algunos casos parecen poseer poblaciones aparentemente numerosas, tal es el caso de Ungla argentina que parece ser relativamente abundante y estar fuertemente asociada a Jordina rhombifolia.

\section{AGRADECIMIENTOS}

Deseamos manifestar nuestro agradecimiento a Flavio N Moschione y a Victoria Massola del Departamento de Áreas protegidas de la Dirección Provincial de Recursos Naturales de la Provincia de Buenos Aires y a Alejandra Silva, Arturo Rosas e Ivan Benoit del Departamento de Patrimonio Silvestre del CONAF por los permisos de muestreos en áreas protegidas que nos fueron concedidos. A Alicia Miravalle del Departamento de Biología, Bioquímica y Farmacia y a Rodrigo Tizón del Departamento de Botánica de la Universidad del Sur en Bahía Blanca por su apoyo y por su ayuda en la identificación de algunas de las plantas aquí citadas, a Juan M. Nieto Nafría de la Universidad de León por identificarnos los pulgones que se citan y a Enrique Garavano por acompañarnos con su mate y su paciencia durante los muestreos.

\section{Referencias}

AdAms, P. A., 1962. Taxonomy of Hawaiian Chrysopa (Neuroptera: Chrysopidae). Proceedings of the Hawaiian Entomological Society, 18(1963): 221- 223.

Adams, P. A., 1967. A Review of the Mesochrysinae and Nothochrysinae (Neuroptera: Chrysopidae). Bulletin of the Museum of Comparative Zoology, 135(4): 215-238.

AdAms, P. A., 1969. New species and synonymy in the genus Meleoma (Neuroptera, Chrysopidae), with a discussion of genitalic homologies. Postilla, 136: 1-18.

Adams, P. A., 1975. Status of the genera Ungla and Mallada Navás (Neuroptera: Chrysopidae). Psyche, 82(2): 167-173.
AdAms, P. A., 1977. Taxonomy of United States Leucochrysa (Neuroptera: Chrysopidae). Psyche, 84(1): 92-102.

Adams, P. A., 1978a. A New Species of Hypochrysa and a New Subgenus and Species of Mallada (Neuroptera: Chrysopidae). Pan Pacific Entomologist, 54: 292-296.

ADAMs, P. A., 1978b. Zoogeography of New World Chrysopidae, a progress report. Folia Entomologica Mexicana, 39-40: 210-211.

Adams, P. A., 1979. A new species of Leucochrysa from Mexico (Neuroptera, Chrysopidae). Folia Entomologica Mexicana, 41: 95-101.

Adams, P. A., 1982a. Ceraeochrysa, a new genus of Chrysopinae (Neuroptera). Neuroptera International, 2(2): 69-75.

ADAms, P. A., 1982b. Plesiochrysopa, a new subgenus of Chrysopa (Neuroptera). Neuroptera International, 2(1): 27-32.

AdAms, P. A., 1983. A New Subespecies of Chrysoperla externa (Hagen) from Cocos Islan, Costa Rica (Neuroptera: Chrysopidae). Bulletin of the Southern California Academy of Sciences, 82(1): 42-45.

Adams, P. A., 1985. Notes on Chrysopodes of the M. N. H. N. in Paris (Neuroptera, Chrysopidae). Revue Française d'Entomologie, N. S., 7(1): 5-8.

AdAms, P. A., 1987. Studies in Neotropical Chrysopidae (Neuroptera). III. Notes on Nodita amazonica Navas and $N$. oenops n. sp. Neuroptera International, 4(4): 287-294.

Adams, P. A. \& Penny, N. A., 1986. Faunal Relations of Amazonian Chrysopidae. In: J. Gepp, H. Aspöck \& H. Hölzel (eds.). Recent Research in Neuropterology. Graz: 119-124.

Adams, P. A. \& Penny, N. A., 1987. Neuroptera of the Amazon Basin, part 11a. Introduction and Chrysopini. Acta Amazonica, 15(3-4): 413-479.

Adams, P. A. \& Penny, N. A., 1992. Review of the South American genera of Nothochrysinae (Insecta: Neuroptera: Chrysopidae). In: M. Canard, H. Aspöck \& M. W. Mansell (eds.). Proceedings of the Fourth International Symposium on Neuropterology. Université Paul-Sabatier. Toulouse: 35-41.

Auad, A. M., Freitas, S. DE \& Barbosa, L. R., 2001a. Influencia de la dieta en la respuesta funcional de Chrysoperla externa (Hagen) (Neuroptera: Chrysopidae) alimentadas con Uroleucon ambrosiae (Thomas) (Hemiptera: Aphididae). Boletín de Sanidad Vegetal, Plagas, 27: 455-463.

Auad, A. M., Freitas, S. DE \& Barbosa, L. R., 2002. Tempo de busca e de manuseio de larvas de Chrysoperla externa (Hagen, 1861) (Neuroptera, Chrysopidae) alimentadas con Uroleucon ambrosiae (Thomas, 1878) (Hemiptera, Aphididae). Revista Brasileira de Entomologia, 46(4): 535-538. 
Auad, A. M., Toscano, L. C., Boiça, A. L. \& Freitas, S. DE, 2001b. Aspectos Biológicos dos Estádios Imaturos de Chrysoperla externa (Hagen) e Ceraeochrysa cincta (Scneider) (Neuroptera: Chrysopidae) Alimentados con Ovos e Ninfas de Bemisia tabaci (Gennadius) Biótipo B (Hemiptera: Aleyrodidae). Neotropical Entomology, 30(3): 429-432.

BANKS, N., 1910. New south american neuropteroid insects. Proceedings of the entomological Society of Washington, 12: 146-160.

Botto, E. N. \& Crouzel, I. S., 1979. Dietas artificiales y capacidad de postura de Chrysopa lanata lanata (Banks) en condiciones de laboratorio. Acta Zoológica Lilloana, 35: 745-758.

Brooks, S. J., 1994. A taxonomic review of the common green lacewing genus Chrysoperla (Neuroptera: Chrysopidae). Bulletin of The Natural History Museum, Entomology Series, 63(2): 137-210.

Brooks, S. J. \& Barnard, P. C., 1990. The green lacewings of the world: a generic review (Neuroptera: Chrysopidae). Bulletin of the British Museum (Natural History), 59(2): 117-286.

Bruch, C., 1917. Desarrollo de Chrysopa lanata Banks (Neuroptera). Physis, 3: 361-369.

CANARD, M., SÉmÉria, Y. \& New, T. R., 1984. Biology of Chrysopidae. Junk Publishers, Series Entomologica, 27. The Hage. 294 pp.

Carvalho, C. F., Souza, B. \& Santos, T. M., 1998. Predation capacity and reproduction potential of Chrysoperla externa (Hagen) (Neuroptera, Chrysopidae) fed on Alabama argillacea (Hübner) eggs. Acta Zoologica Fennica, 209: 83-86.

Crouzel, I. S. \& Botto, E. N., 1976. Ciclo de vida de Chrysopa lanata (Banks) y algunas observaciones biológicas en condiciones de laboratorio. Revista de Investigación Agropecuaria INTA, ser. 5, Patología Vegetal, 13(1): 1-14.

Crouzel, I. S. DE \& SAINI, E., 1979. Llave dilematica para el reconocimiento de los tres estadios larvales de Chrysopa lanata lanata (Banks) (Neurop. Chrysopidae). Acta Zoológica Lilloana, 35: 417-425.

ERIZE, F., 2000a. Especies forestales de la Argentina occidental I. El Ateneo. Buenos Aires. 120 pp.

ERIzE, F., 2000b. Especies forestales de la Argentina oriental II. El Ateneo. Buenos Aires. 124 pp.

Esben-Petersen, P., 1924. More Neuroptera from Juan Fernandez and Easter Island. Natural History of Juan Fernandez and Estern Island, 3: 309-313.

Figueira, L. K., Lara, F. M. \& CruZ, I., 2002. Efeito de Genótipos de Sorgo Sobre o Predador Chrysoperla externa (Hagen) (Neuroptera: Chrysopidae) Alimentado com Schizaphis graminum (Rondan) (Hemip-tera: Aphididae). Neotropical Entomology, 31(1): 133-139.

FreitAs, S. DE, 2003. Chrysoperla Steinman, 1964 (Neuroptera, Chrysopidae): descrição de uma nova espécie do Brasil. Revista Brasileira de Entomología, 47(3): 385-387.

Freitas, S. DE \& Fernández, O. A., 1992. A preliminary statement on green lacewings in citrus in the Jaboticabal region of Brazil. (Insecta: Neuroptera: Chrysopidae). Proceedings of the Fourth International Symposium on Neuropterology. Canard, M., Aspöck, H. \& Mansell, M. W. Eds., Toulouse: 147-150.

Freitas, S. DE \& Penny, N. D., 2001a. Two New Genera of Neotropical Chrysipini (Neuroptera: Chrysopidae). Journal of Kansas Entomological Society, 73(3): 164170.

Freitas, S. DE \& Penny, N. D., 2001b. The Green Lacewings (Neuroptera: Chrysopidae) of Brazilian Agro-ecosistems. Proceedings of the California Academy of Sciences, 52(19): 245-395.

Gitirana Neto, J. G., Carvalho, C. F., Souza, B. \& Santa Cecilia, L. V. C., 2001. Flutiação populacional de espécies de Ceraeochrysa Adams, 1982 (Neuroptera: Chrysopidae) em citros, na Região de Lavras-Mg. Ciencia e Agrotecnologia, 25(3): 550559.

GonzÁlez Olazo, E. V., 1987. Los neurópteros asociados con los cultivos cítricos de la provincia de Tucumán y descripción de una nueva especie de Nomerobius (Hemerobiidae). Cirpon, Revista de Investigación, 5(1-4): 37-54.

GonzÁlez Olazo, E. V., 1996. Catálogo de los tipos de Neuroptera (Insecta: Holometabola) depositados en colecciones argentinas. Acta Zoológica Lilloana, 43(2): 373-391.

Haene, E. \& Gostaro, A., 2001. 100 árboles argentinos. Albatros. Buenos Aires. 189 pp.

Hagen, H. A., 1861. Synopsis of the Neuroptera of North America. With a list of the South American species. Smithsonian Miscellaneous Collections, 4: $1-347$.

Hoffmann, A. E., 1997. Flora silvestre de Chile. Fundación Claudio Gay. Santiago. 253 pp.

Kimmins, D. E., 1952. A revision of the Genera of the Apochrysinae. Annals and Magazine of Natural History, 12(5): 929-944.

McEwen, P., New, T. R. \& Whittington, A. E., 2001. Lacewings in the Crop Environment. Cambridge University Press. Cambridge. 546 pp.

Monserrat, V. J., 2003. Contribución al conocimiento de los hemeróbidos de Patagonia y Tierra del Fuego (Insecta, Neuroptera: Hemerobiidae). Graellsia, 59(1): 37-56.

Monserrat, V. J., 2005. New data on the dusty wings from Coquimbo, Patagonia and Tierra del Fuego (Neuroptera, Coniopterygidae). Entomofauna, 26: $1-13$.

Muma, M. H., 1959. Chrysopidae associated with citrus in Florida. Florida Entomologist, 42: 21-29. 
NAVÁs, L., 1910a. Nueva especie de Neuróptero. Revista Chilena de Historia Natural, 14: 70.

NAvÁs, L., 1910b. Crisópidos (Ins. Neur.) nuevos. Brotéria, Série Biologia, 9: 38-59.

NAVÁs, L., 1910c. Neurópteros chilenos (1 ${ }^{\mathrm{a}}$ serie). Revista Chilena de Historia Natural, 14: 235-241.

NavÁs, L., 1911. Chrysopes noveaux. Annales de la Societé Scientifique de Bruxelles, 35: 258-279.

NAVÁs, L., 1913. Crisópidos sudamericanos. Brotéria, Série Biologia, 11: 73-104, 149-168.

NAVÁs, L., 1914. Neurópteros sudamericanos. Primera serie. Brotéria, Série Biologia, 12: 45-56, 215-234.

NAVÁs, L., 1915. Neurópteros nuevos o poco conocidos VI. Memorias de la Real Academia de Ciencias y Artes de Barcelona, 12: 119-136.

NAVÁs, L., 1917a. Algunos insectos Neurópteros de la Argentina. Physis, 3: 186-196.

NavÁs, L., 1917b. Algunos insectos de la República Argentina. Revista de la Real Academia de Ciencias de Madrid, 16: 491-504.

NAVÁs, L., 1919a. Algunos insectos de la Repúbica Argentina. Revista de la Real Academia de Ciencias de Madrid, 17: 287-305.

NAVÁs, L., 1919b. Insecta nova V, VI, VII. Memorie della Accademia pontificia dei Nuovi Lincei, 2(5): 110, 11-29.

NAVÁs, L., 1920. Insectos sudamericanos $1^{\mathrm{a}}, 2^{\mathrm{a}}, 3^{\mathrm{a}}$ series. Anales de la Sociedad Cientifica Argentina, 90: 33-72.

NAVÁs, L., 1921a. Insectos sudamericanos $4^{\mathrm{a}}$ serie. Revista de la Real Academia de Ciencias de Madrid, 19: 255-267.

NavÁs, L., 1921b. Algunos insectos de Santa Fe (República Argentina). Estudios, 21: 49-53.

NAVÁs, L., 1922a. Estudis sobre Neurópters. Arxius del Institut des Ciences, 7: 179-203.

NAVÁs, L., 1922b. Insectos de la Argentina y Chile. Estudios, 22: 358-368.

NAVÁs, L., 1922c. Algunos insectos del Museo de París. Revista de la Academia de Ciencias de Zaragoza, 7 : 15-51.

NaVÁs, L., 1923. Crisópidos (Ins. Neur.) neotrópicos. Revista Chilena de Historia Natural, 27: 110-116.

NAVÁs, L., 1925a. Insectos neotrópicos $1^{a}$ serie. Revista Chilena de Historia Natural, 29: 305-113.

NAVÁs, L., 1925b. Neurópteros del Museo de Berlin. Revista de la Academia de Ciencias de Zaragoza, 9: 20-34.

NAVÁs, L., 1926. Insectos de la Argentina y Chile. $2^{\mathrm{a}}$ serie. Estudios, 31: 103-111.

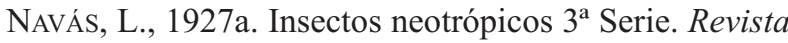
Chilena de Historia Natural, 31: 316-328.

NAVÁs, L., 1927b. Insectos del Museo de París, $4^{\mathrm{a}}$ serie. Brotéria, Série Zoologia, 24(1): 5-33.
NaVÁs, L., 1928. Insectos del Museo de Hamburgo. Boletín de la Sociedad entomológica Española, 11: 59-67, 90-100, 121-138, 165.

NAVÁs, L., 1929a. Insectos neotrópicos, $5^{\mathrm{a}}$ serie. Revista Chilena de Historia Natural, 33: 17-24.

NAVÁs, L., 1929b. Insectos de la Argentina. Quinta serie. Revista de la Sociedad entomológica Argentina, 10: 219-225.

NAVÁs, L., 1929c. Insectos exóticos neurópteros y afines del Museo Civico de Génova. Annali di Museo Civico di Storia Naturale Giacono Doria, 53: 354-389.

NAVÁs, L., 1930. Insectos neotrópicos. Sexta serie. Revista Chilena de Historia Natural, 34: 62-75.

NAVÁs, L., 1932a. Alcuni Insetti del Museo di Zoologia della R. Universita di Torino. Bollettino dei Musei di Zoologia e di Anatomia Conparata della Reale Università di Torino, serie III, 42(26): 1-38.

NAVÁs, L., 1932b. Insectos de la Argentina y Chile. Revista de la Sociedad entomológica Argentina, 22(5): 79-86.

NAVÁs, L., 1933. Insectos sudamericanos. $7^{\mathrm{a}}$ Ser. Revista de la Real Academia de Ciencias de Madrid, 30: 303-314.

Penny, N. D., 1977. Lista de Megaloptera, Neuroptera e Raphidioptera do México, América Central, ilhas Caraíbas e América do Sul. Acta Amazonica, 7(4): $1-61$.

PENNY, N. D., 1996. A Revision of Parachrysopiella (Neuroptera: Chrysopidae). Journal of the Kansas Entomological Society, 69(3): 222-233.

Penny, N. D., 1997. Four new species of Costa Rican Ceraeochrysa (Neuroptera: Chrysopidae). Pan Pacific Entomologist, 73(2): 61-69.

Penny, N. (Ed.), 2002. A Guide to the Lacewings (Neuroptera) of Costa Rica. Proceedings of the California Academy of Sciences, 53(12): 161-457.

Penny, N. \& Lee, V. F., 1996. Types of Mecoptera, Megaloptera, Raphidioptera, and Neuroptera (Insecta) in the collection of the California Academy of Sciences. Proceedings of the California Academy of Sciences, 49(4): 127-149.

Penny, N. D. \& Freitas, S. DE, 2001. Sexual Dimorphism in Ceraeochrysa cubana (Neuroptera: Chrysopidae). Journal of the Kansas Entomological Society, 74(1): 57-58.

Pérez Gelabert, D. E. \& Flint, O. S., 2001. Annotated List of the Neuroptera of Hispaniola, with New Faunnistic Records of some Species. Journal of Neuropterology, 3: 9-23.

Ru, N., Whitconb, W. H., Murphey, M. \& Carlysle, T. C., 1975. Biology of Chrysopa lanata (Neuroptera: Chrysopidae). Annals of the Entomological Society of America, 68: 187-90.

StAnge, L. A., 1967. Catálogo de Neuroptera de Argentina y Uruguay. Acta Zoológica Lilloana, 22: 1-87. 
TAuber, C. A., 1974. Systematics of north american Chrysopid larvae: Chrysopa carnea group (Neuroptera). Canadian Entomologist, 106: 1133-1153.

Tauber, C. A., León, T. De, López Arroyo, J. I. \& TAUBer, J. M., 1998. Ceraeochrysa placita (Neuroptera: Chrysopidae): Generic Characteristics of Larvae, Larval Description, and Life Cicle. Annals of the Entomological Society of America, 91(5): 608-618.

TJeder, B., 1966. Neuroptera-Planipennia. The lacewing of Southern Africa 5. Family Chrysopidae. South African Animal Life, vol. 8. Swedish Natural Science Research Council. B. Hanström, P. Brinck. \& G. Rudebec (eds.). Stockholm: 296-408.

TJEDER, B., 1971. Redescriptions and lectotype designations of some Exotic Chrysopidae (Neur.). Entomologica Scandinavica, 2: 110-118.
WiLliams, F. X., 1931. Family Chrysopidae (The Green Lace-wings). In: Neuroptera Handbook of the Insects and other Invertebrates of Hawaiian Sugar Cane Fields. Bulletin of the Hawaiian Sugar Planters' Association Experiment Station (Entomological Series), Honolulu: 130-132.

Zimmerman, E. C., 1957. Chrysopidae. In: Insects of Hawaii, vol 6. Ephemeroptera, Neuroptera, Trichoptera. University of Hawaii Press, Honolulu: 86-152.

Recibido, 8-VI-2005 Aceptado, 2-XI-2005 Publicado, 31-XII-2005 